\title{
Characteristics of electron cyclotron waves creating field-aligned and transverse plasma potential structures
}

\author{
K Takahashi ${ }^{1,3}$, T Kaneko ${ }^{1}$, R Hatakeyama ${ }^{1}$ and A Fukuyama ${ }^{2}$ \\ ${ }^{1}$ Department of Electronic Engineering, Tohoku University, Sendai 980-8579, Japan \\ ${ }^{2}$ Department of Nuclear Engineering, Kyoto University, Kyoto, 606-8501, Japan \\ ${ }^{3}$ Department of Electrical and Electronic Engineering, Iwate University, Morioka \\ 020-8551, Japan
}

E-mail: kazunori@iwate-u.ac.jp

\begin{abstract}
Characteristics of electromagnetic waves of azimuthal mode number $m= \pm 1$ are investigated experimentally, analytically, and numerically when the waves triggering the field-aligned and transverse plasma-potential structure modification near an electron cyclotron resonance (ECR) point are injected into an inhomogeneously magnetized plasma with high-speed ion flow. The waves of $m=+1$ and -1 modes generate an electric double layer near the ECR point at the radially central and peripheral areas of the plasma column, respectively, and the transverse electric fields are consequently formed. At these areas the waves have a right-handed polarization and are absorbed through the ECR mechanism, where the experimental and analytical results do show the polarization reversal along the radial axis. The numerical results by Plasma Analysis by Finite element method (FEM)/ Wave analysis by FEM (PAF/WF) code show that the wave-absorption area is localized at the radially central and peripheral areas for $m=+1$ and -1 mode waves, respectively, being consistent with the experimental and analytical ones.
\end{abstract}




\section{Introduction}

Studies on the formation of plasma-potential structures have been advanced in connection with the behaviors of charged particles in space [1, 2], fusion-oriented plasmas [3], and electric propulsion devices [4, 5, 6], which are divided into two types of field-aligned and transverse structures, i.e., parallel and perpendicular electric fields in magnetized plasmas. Regarding the parallel electric fields, the investigation on electric double layers (DLs) has especially been one of the interesting topics, which has been focused on clarifying the mechanisms of the particle acceleration in auroral zone by using laboratory plasmas $[7,8,9]$. After that direct observations of the parallel electric field in a space plasma give rise to up-to-date argument about the DL $[2,10]$. Recently current-free DLs in magnetically expanding plasmas have been vigorously studied for the purpose of applying to an electric thruster [4, 5, 6, 11, 12]. The parallel electric field is also utilized for the confinement of energetic ions in a tandem mirror fusion machine $[3,13]$. The perpendicular electric fields, i.e., the radially sheared electric fields have also been studied because it is well known to suppress plasma instabilities and turbulence driving particle transports $[14,15]$. Moreover, the effects of the perpendicular electric fields in magnetically expanding plasmas on the divergence of ion beams are also now discussed [16].

In association with the above-mentioned subjects, potential structures modified by electron cyclotron resonance (ECR) heating in laboratory plasmas have been investigated for the case that microwaves are injected along inhomogeneous magneticfield lines $[17,18,19]$. The subsequent series of the experiments demonstrated the detailed relations between the potential height of the modified structure and the fieldaligned ion flow energy [19]. In addition, the relation between the two-dimensional structures and the propagation of the electromagnetic waves relating to ECR has briefly been reported by authors [18]. These two issues are significant and related to essential physics of the potential creation using elaborate ECR heating in the tandem mirror machine.

The present paper reveals the detailed experimental, analytical, and numerical results on the electromagnetic waves of azimuthal mode number $m= \pm 1$, which can form the parallel electric fields due to the generation of the DL at the radially different areas of the plasma column depending on the wave mode number, and the resultant localized perpendicular electric fields.

\section{Experimental setup}

Experiments are performed in $Q_{T}$-Upgrade machine of Tohoku University, as shown in Fig. 1(a). The machine has a 450-cm-long and 20.8-cm-diameter stainless steel vacuum chamber evacuated to a base pressure below $10^{-4} \mathrm{~Pa}$ by two turbomolecular/rotary pumping systems. The converging magnetic-field configuration of types (I) and (II) presented in Fig. 1(b) as solid lines can be applied by two parties of the solenoidal coils, 
where $z=0$ and each axis $(x, y, z)$ are defined as the axial center of the vacuum chamber and as indicated at the upper left of Fig. 1(a). The dashed line in Fig. 1(b) presents the ECR magnetic-field strength $(2.14 \mathrm{kG})$ of $6 \mathrm{GHz}$ microwave, and the ECR points are indicated by arrows at $z=26$ and $45 \mathrm{~cm}$ for the configurations (I) and (II), respectively. A plasma source providing a control of the field-aligned ion flow energy $[20,21]$ is set at the low magnetic-field side. Briefly, argon ions in an upstream plasma are created by a direct current discharge between an oxide cathode and a mesh anode, where the plasma potential of the upstream plasma is proportional to the anode potential $V_{a}$. An electron reflector biased at $-120 \mathrm{~V}$ and an electron emitter of mesh shape, which is called mesh emitter, are located in front of the anode; the ions accelerated by the potential difference between the upstream plasma and the downstream side of the mesh emitter can be neutralized by the thermoionic electrons emitted from the mesh emitter. Since the plasma potential of the downstream plasma is decided by the potential of the mesh emitter supplying the electrons to the downstream plasma, the field-aligned ion flow with energy corresponding to the potential difference between the upstream and downstream plasmas can be generated and its energy can be controlled by the anode potential $V_{a}$. The argon gas is introduced from the chamber sideport near the source and the plasma source is operated at low gas pressures of about $10 \mathrm{mPa}$. The anode potential $V_{a}$ is set at $15 \mathrm{~V}$ in the present experiments and the plasma column is terminated by an insulator plate. The plasma radius is limited to about $3 \mathrm{~cm}$ by a limiter located at the same position as the anode. Under these conditions, the retarding field energy analyzer [21] detects only the flowing ions in the downstream plasma, at the collector bias voltage of about 16-17 V corresponding to the potential of the upstream plasma. An electron density measured in the downstream plasma is about $10^{10} \mathrm{~cm}^{-3}$.

Microwave (frequency: $\omega / 2 \pi=6 \mathrm{GHz}$ ) with $m=+1$ and -1 modes are selectively launched from the high magnetic-field side through a horn antenna with a dielectric polarizer. The wave transmitted through a rectangular waveguide as rectangular $\mathrm{TE}_{01}$ mode from the microwave power supply, is converted into circular $\mathrm{TE}_{11}$ mode and is polarized by the dielectric polarizer into $m=+1$ or -1 modes, where the radius of the circular waveguide is $1.5 \mathrm{~cm}$. The characteristics of the dielectric polarizer are experimentally investigated; the axial ratio of the polarized waves radiated from the antenna is about 1.1. Electric fields $E_{x}, E_{y}$, and $E_{z}$ of the microwaves are measured using movable dipole antennas [22]. In the experiments on the creation of the plasmapotential structures, a klystron amplifier is used for $50 \mathrm{~W}$ microwave injections. When the wave propagation is experimentally investigated, wave measurements are performed under the condition that the wave does not change the plasma parameter, which are carried out using a network analyzer. The amplitude and the phase of the detected wave can be obtained from the complex transmission coefficient $S_{21}$ between the horn antenna and the dipole antenna. An argument $\angle S_{21}$ and an absolute value $\left|S_{21}\right|$ are equivalent to the phase difference between the detected wave field and the reference signal, and the wave amplitude. Spatial profiles of the plasma parameters are measured by two single-tipped Langmuir probes, which are movable in the $x$ - $z$ plane at $y=0$ and 
in the $x$-y plane at $z=26 \mathrm{~cm}$, respectively. The local plasma potential is obtained from the current-voltage curve through the classical analysis.

Figure 1(c) shows the axial profiles of the plasma potential $\phi_{p}$ at the radial center of the plasma column, for microwave power $P_{i n}=0 \mathrm{~W}$ (open circles) under the configuration (I), and for $P_{i n}=50 \mathrm{~W}$ of $m=+1$ mode under configurations (I) (closed circles) and (II) (open triangles), all of which are measured at $30 \mu \mathrm{sec}$ after the wave injection. $\phi_{p}$ is axially homogeneous for the case of $P_{i n}=0 \mathrm{~W}$, while a localized potential jump corresponding to the ion flow energy, i.e., a DL, is clearly observed near the ECR points of $z=26 \mathrm{~cm}$ and $z=45 \mathrm{~cm}$ for the configurations (I) and (II), respectively, as previously reported by authors [19]. The measured electron temperature without microwave injection is spatially uniform and is about $0.3 \mathrm{eV}$. With microwave injection, on the other hand, the measured electron temperature is about $8 \mathrm{eV}$ and $2 \mathrm{eV}$ in the high- and low-potential sides, respectively. We confirmed that the observed potential structures are not due to the temperature gradient and density gradient, through an analysis of the force applied to the electrons. The detailed mechanisms of the DL generation has already been reported in Ref. [19]. Simply stated here, a magneticmirror effect, i.e., $-\mu \nabla B$ force, is markedly enhanced by ECR heating and reflects a part of electrons; this effect and the inertia of the flowing ions bring about a chargeseparation phenomenon. This is so called the electric double layer, and its potential height is self-consistently determined for satisfying the charge neutrality by reflecting a part of energetic ions flowing along the magnetic-field lines.

\section{Dispersion analysis}

We consider the azimuthally and radially uniform plasma column. Dispersion analysis in the bounded plasma has already described [22, 23, 24]. Briefly, a dispersion relation of electromagnetic waves in a cold plasma is derived by Maxwell' sequation as [23]

$$
\left(\gamma^{2}+\kappa_{2}^{2}+\gamma k_{\perp}^{2}\right) \kappa_{3}+k_{\perp}^{2}\left[\kappa_{1}\left(\gamma+k_{\perp}^{2}\right)-\kappa_{2}^{2}\right]=0,
$$

where $\gamma \equiv k_{\|}^{2}-\kappa_{1}$, and $k_{\|}$and $k_{\perp}$ are the wavenumbers parallel and perpendicular to a static magnetic field $\mathbf{B}$, respectively; $\kappa_{1}, \kappa_{2}$, and $\kappa_{3}$ are the dielectric tensor elements multiplied by $k_{0}^{2}$, followed by Swanson notation [23]. When the electric fields are assumed to propagate toward the $z$ direction, the wave fields of azimuthal mode number $m$ can be represented by

$$
\mathbf{E}=\mathbf{E}(r) \exp \left[i\left(k_{\|} z+m \theta-\omega t\right)\right],
$$

where the components of the electric-field vector $\mathbf{E}(r)$ are derived as

$$
\begin{aligned}
& E_{z}(r)=\frac{\omega \kappa_{1} \beta A}{k_{\|} \kappa_{2} \kappa_{3}} J_{m}\left(k_{\perp} r\right), \\
& E_{r}(r)=\frac{i \omega \delta A}{\kappa_{2} k_{\perp}} J_{m}^{\prime}\left(k_{\perp} r\right)-\frac{m \omega A}{r k_{\perp}^{2}} J_{m}\left(k_{\perp} r\right), \\
& E_{\theta}(r)=-\frac{m \omega \delta A}{r \kappa_{2} k_{\perp}^{2}} J_{m}\left(k_{\perp} r\right)-\frac{i \omega A}{k_{\perp}} J_{m}^{\prime}\left(k_{\perp} r\right) .
\end{aligned}
$$


Here $\beta \equiv \gamma-\kappa_{2}^{2} / \kappa_{1}+k_{\perp}^{2}, \delta \equiv \gamma+k_{\perp}^{2}$, and $A$ and $J_{m}$ are an amplitude constant and Bessel function of order $m$, respectively. $E_{r}$ and $E_{\theta}$ in cylindrical coordinates correspond to experimentally obtained $E_{x}$ and $E_{y}$ in rectangular coordinates. Although the radial boundary conditions restrict the perpendicular wavenumber $k_{\perp}$, the introduction of the boundary conditions complicates the dispersion analysis. For simplification, the perpendicular wavenumber experimentally estimated from the radial profile of the electric field $E_{z}$ is used for the calculation of parallel wavenumber $k_{\|}$. This approach can introduce the effects of the boundary conditions and has actually explained the propagation of $m=0$ mode wave in our previous studies [22,24]. Although the parallel wavenumber $k_{\|}$is also affected by axial boundary conditions, we can neglect its effects because the parallel wavelength is much less than the axial length of the plasma column in our experimental configurations. Moreover, effects of the inhomogeneous magneticfield configuration on the plasma density and the plasma radius should be included in the above-mentioned dispersion theory, which has already been described in Ref. [24].

It is well known that the wave polarization plays important roles in the ECR phenomenon. The above dispersion relation and electric-field components derive a polarization index $S$ as

$$
S \equiv \frac{\left|E_{r}+i E_{\theta}\right|}{\left|E_{r}-i E_{\theta}\right|}
$$

Here, $0<S<1$ and $1<S<\infty$ represent right-handed and left-handed polarizations, and $S=0, S=1$, and $S=\infty$ correspond to circularly right-handed, linear, and circularly left-handed polarizations, respectively.

\section{Experimental and analytical results}

Figure 2 gives two dimensional profiles of the plasma potential $\phi_{p}$ in the $x-z$ and $x-y$ planes for $\left[(\mathrm{a})\right.$ and (b)] $P_{\text {in }}=0 \mathrm{~W}$, for $[(\mathrm{c})$ and (d) $] P_{i n}=50 \mathrm{~W}$ of $m=+1$ mode, and for $[(\mathrm{e})$ and $(\mathrm{f})] P_{i n}=50 \mathrm{~W}$ of $m=-1$ mode. Here the $x-z$ plane measurements were carried out under the magnetic-field configuration (I), while the $x-y$ plane measurements were performed under the configuration (II) because the Langmuir probe movable in $x$ - $y$ plane was required to be set at the high-potential side. $\phi_{p}$ for $P_{i n}=0 \mathrm{~W}$ is spatially uniform as shown in Figs. 2(a) and 2(b). In the cases of $m=+1$ and -1 mode wave injections, shown in Figs. 2(c)-2(f), the DL structure, i.e., the field-aligned potential jump, is found to be generated at the central and peripheral areas of the plasma column, respectively. The selective generation of the DL at the central and peripheral areas can create the radial potential structures in the high-potential side as presented in Figs. 2(c)-2(f). These results suggest that the potential structure in the radial direction can actively be controlled by just selecting the azimuthal mode number $m$ of the microwave; it is clearly demonstrated that the radially outward and inward electric fields are formed in the high-potential side for $m=+1$ and -1 modes, respectively.

In order to understand the above-mentioned difference in the potential structures created by $m=+1$ and -1 mode waves, the propagations of the microwaves have to 
be investigated. As mentioned in the Sec. 3, the radial profile of the axial component $E_{z}$ of the wave electric field can be expressed by the combination of Eqs. (2) and (3). According to these equations, the phase difference in $E_{z}$ for $x>0$ and $x<0$, i.e., $\theta=0$ and $\theta=180$ degree, is 180 degree and the radial profile of the axial component $\left|E_{z}\right|$ of the electric-field amplitude can be expressed by the Bessel function of order one for $m= \pm 1$. Here, $\theta$ is the azimuthal position, and $\theta=0$ and $\theta=180$ degree correspond to $x>0$ and $y=0$, and $x<0$ and $y=0$. The measured argument $\angle S_{21}$ and absolute value $\left|S_{21}\right|$ of the complex transmission coefficient between the horn antenna and the dipole antenna which is immersed in the plasma and detects the axial electric field $E_{z}$, are plotted in Figs. 3(a) and 3(b) as closed circles for $m=+1$ and -1 mode, respectively. Here, $\angle S_{21}$ and $\left|S_{21}\right|$ correspond to the phase difference between the detected and reference signals, and the electric-field strength $\left|E_{z}\right|$ of the waves. The solid lines in Figs. 3(a) and 3(b) are the absolute value of the Bessel functions $\left|J_{1}(1.02 r)\right|$ and $\left|J_{1}(1.20 r)\right|$, respectively, which are the curve fitting for the experimental results. It is found that the phase differences between $x>0$ and $x<0$ are clearly 180 degree and the radial profiles of $\left|S_{21}\right|$, i.e., $\left|E_{z}\right|$, can be represented by the Bessel function of order one in both Figs. 3(a) and 3(b). Thus, the excited wave modes are surely $m=+1$ and -1 , and the perpendicular wavenumber $k_{\perp}$ can be estimated as $k_{\perp} \sim 1.02 \mathrm{~cm}^{-1}$ for $m=+1$ mode, and $\sim 1.20 \mathrm{~cm}^{-1}$ for $m=-1$ mode.

Substituting the observed perpendicular wavenumber for $k_{\perp}$ in the dispersion relation given by Eq. (1), we can analytically obtain the parallel wavenumber $k_{\|}$. The magnetic-field strength is regarded as a variable in the calculation of the dispersion relation, since the wave frequency $\omega / 2 \pi$ is fixed at $6 \mathrm{GHz}$ and the local magnetic-field strength, i.e., the local electron cyclotron resonance frequency $\omega_{c e} / 2 \pi$ is varied when we consider the spatial wave propagation in $z$ direction. The calculated results for $k_{\perp}=1.02$ $\mathrm{cm}^{-1}$ and $1.20 \mathrm{~cm}^{-1}$, i.e., for $m=+1$ and -1 , are plotted as solid lines in Figs. 4(a) and $4(\mathrm{~b})$, respectively. The parallel wavenumbers $k_{\|}$measured at various $z$ position are also plotted as closed circles in Fig. 4. It is found that the waves propagating in our experiments are the slow waves, where the slow and fast waves are defined as the dispersion branches with slower and faster phase velocities for $\omega / \omega_{c e}<1$. In Figs. 4(a) and $4(\mathrm{~b})$, it is found that the slow wave is resonant at ECR point, while the fast wave has no resonance.

The wave polarization would play an important role in the ECR heating which creates the plasma potential structures shown in Fig. 2, where $x$ profiles of a phase difference $\Delta \varphi$ between $E_{x}$ and $E_{y}$ of the wave electric fields for $m=+1$ and -1 modes are measured at $z=15 \mathrm{~cm}$ under the configuration (I) and are plotted in Figs. 5(a) and 5(b) as open circles, respectively. We can identify the polarization direction from $\Delta \varphi$; the right- and left-handed polarizations are represented by $-180^{\circ}<\Delta \varphi<0^{\circ}$ and $0^{\circ}<\Delta \varphi<180^{\circ}$, respectively. It is found in Fig. 5(a) that the polarization for $m=+1$ is right handed around the central area and left handed around the peripheral area, i.e., the polarization is reversed along the radial axis. In contrast, the left- and righthanded polarizations are observed around the central and peripheral areas for the case 
of $m=-1$, as plotted in Fig. 5(b). The theoretical polarization index $S$ is compared with the experimentally observed polarization direction, where $S$ is derived from the dispersion relation and the electric fields of the slow waves. The results are shown in Figs. 5(a) and 5(b) as solid lines. Once again, the right- and left-handed polarizations correspond to $S<1$ and $S>1$, respectively. The analytically obtained $S$ also shows that the wave polarization for $m=+1(m=-1)$ mode is right handed (left handed) around the central area and left handed (right handed) around the peripheral area, i.e., the observed polarization profiles are well explained by the dispersion theory in bounded plasmas. We can deduce that the wave is absorbed and the DL is generated around the area where the polarization is right handed. As the right-handedly polarized electric field would continue to accelerate the gyratory electrons, the wave is considered to be absorbed through the collisionless cyclotron damping mechanisms. As a result, the radial location of the DL can be changed by selecting $m$; it is experimentally demonstrated that the direction of the transverse electric field can be changed by the microwaveantenna operation. In this experiments, the observation of the wave absorption profiles was difficult because we cannot distinguish whether the waves are absorbed or reflected at the ECR point, from the wave power profiles. Spatial profiles of the absorbed wave power are shown in the next section through numerical analysis.

\section{Numerical results}

The wave propagation in the inhomogeneously magnetized plasmas near the ECR point is numerically investigated by Plasma Analysis by Finite element method (FEM)/ Wave analysis by FEM (PAF/WF) code developed by Fukuyama [25]. This code solves Maxwell's equation with a cold plasma dielectric tensor including collisional damping as a boundary-value problem.

The plasma density is assumed to be azimuthally uniform, which allows us to analyze in two dimension of $r-z$ plane. Figures 6(a)-6(c) show the axial $(z)$ profile of the external magnetic field, the radial $(r)$ profiles of the plasma density at $z=-50$ and $-120 \mathrm{~cm}$, and the $r-z$ profile of the plasma density, where the plasma density is set at $10^{10} \mathrm{~cm}^{-3}$ same as the experimental condition, and the ECR point of the $6 \mathrm{GHz}$ wave is $z=-68 \mathrm{~cm}$. It is found that the plasma radius is varied by the nonuniform magnetic-field lines. A 1.5-cm-radius one-turn loop antenna is set at $z=-159 \mathrm{~cm}$, which corresponds to the radius of the circular waveguide in the experiment. At the radially outer side $(r=10 \mathrm{~cm})$ and the axially downstream side $(z=0 \mathrm{~cm})$ of the wave propagation, the grounded metal is located as boundary conditions similar to the experimental boundaries. Under these conditions, the spatial profiles of the wave power absorbed into the plasma are numerically obtained for $m= \pm 1$ modes.

At first, in order to have a confidence in the relation between the experiments and the numerical code, the radial profiles of the axial component $E_{z}$ of the wave electric field for $m=+1$ and $m=-1$ modes are shown in Fig. 7(a) and 7(b), respectively. The numerical results are found to resemble the experimental ones shown in Fig. 3, where 
zero crossing points of $E_{z}$ are about $r=4.2 \mathrm{~cm}$ for $m=+1$ and $r=4 \mathrm{~cm}$ for $m=-1$, being very close to the results in Fig. 3 .

Figure 8 shows the $r-z$ profiles of the wave power $P_{a b s}$ absorbed into the plasma for (a) $m=+1$ and (b) $m=-1$ modes, which are relevant to the electron-heating profiles observed in Fig. 2. Near the ECR point at $z=-68 \mathrm{~cm}$, it is found the $m=+1$ mode wave is absorbed around the central area of the plasma column, while the absorption area of the $m=-1$ mode wave is localized around $r \sim 1.5 \mathrm{~cm}$. In the numerical investigation using $\mathrm{PAF} / \mathrm{WF}$ code, the power absorption is due to the collisional damping. The numerical results imply that the wave is absorbed through the collisional damping mechanism, while the experimental and analytical results suggest the collisionless cyclotron damping as mentioned in Sec. 4. Comprehensively considering the experimental, analytical, and numerical results, the $m=+1$ and -1 mode waves are absorbed through both the collisional damping and collisionless cyclotron damping at the radially central and peripheral areas where the polarization is right handed, respectively, which is due to the polarization reversal along the radial axis. For quantitative estimation of the wave power absorption through the collisional and collisionless damping mechanisms, a kinetic analysis is required.

\section{Conclusion}

The propagation and absorption of the $m= \pm 1$ mode electromagnetic waves near the ECR point in an inhomogeneously magnetized plasma, which generate the DL at the radial center and periphery of the plasma column, are investigated experimentally, analytically, and numerically. It is found that the radial location of the DL is in good agreement with the region where wave is absorbed. As a result of the selective generation of the DL, the radially inward and outward transverse electric fields are formed. The wave absorption profile is dominated by the polarization profile, where the polarization is reversed along the radial axis, and is right-handed (left-handed) at the center and left-handed (right-handed) at the periphery for $m=+1(m=-1)$ modes. The results would lead to providing a new method of the plasma structure control by the waves with ECR frequency.

\section{Acknowledgments}

The authors are indebted to H. Ishida for his technical assistance. We also express our gratitude to Professor K. Sawaya, Professor Q. Chen, and Dr. H. Sato for their useful comments for design of the microwave antenna. This work was supported by the Japan Society for the Promotion of Science for Young Scientists. 


\section{References}

[1] Mozer F S, Carlson C W, Hudson M K, Torbert R B, Parady B, Yatteau J and Kelley M C 1977 Phys. Rev. Lett. 38292

[2] Ergun R E, Su Y -J, Andersson L, Carlson C W, McFadden J P, Mozer F S, Newman D L, Goldman M V and Strangeway R J 2001 Phys. Rev. Lett. 87045003

[3] Inutake M, Cho T, Ichimura M, Ishii K, Itakura A, Katanuma I, Kiwamoto Y, Kusama Y, Mase A, Miyoshi S, Nakashima Y, Saito T, Sakasai A, Sawada K, Wakaida I, Yamaguchi N and Yatsu K 1985 Phys. Rev. Lett. 55939

[4] Charles C 2007 Plasma Sources Sci. Technol. 16 R1, and references therein

[5] Charles C and Boswell R W 2007 Appl. Phys. Lett. 91201505

[6] Takahashi K, Oguni K, Yamada H and Fujiwara T 2008 Phys. Plasmas 15084501

[7] Quon B H and Wong A Y 1976 Phys. Rev. Lett. 371393

[8] Stenzel R L, Ooyama M and Nakamura Y 1980 Phys. Rev. Lett. 451498

[9] Sato N, Hatakeyama R, Iizuka S, Mieno T, Saeki K, Rasmussen J J and Michelsen P 1981 Phys. Rev. Lett. 461330

[10] Ergun R E, Andersson L, Main D S, Su Y -J, Carlson C W, McFadden J P and Mozer F S 2002 Phys. Plasmas 93685

[11] Takahashi K, Charles C, Boswell R W, Kaneko T and Hatakeyama R 2007 Phys. Plasmas 14 114503

[12] West M D, Charles C and Boswell R W 2008 J. Propul. Power 24134

[13] Cho T, Higaki H, Hirata M, Hojo H, Ichimura M, Ishii K, Itakura A, Katanuma I, Kohagura J, Nakashima Y, Saito T, Tatematsu Y, Yoshikawa M, Minami R, Numakura T, Yoshida M, Watanabe H, Yatsu K and Miyoshi S 2003 Nucl. Fusion 43293

[14] Groebner R J, Burrell K H and Seraydarian R P 1990 Phys. Rev. Lett. 643015

[15] Hatakeyama R and Kaneko T 2004 Phys. Scripta T107 200

[16] Takahashi K and Fujiwara T 2009 Appl. Phys. Lett. 94061502

[17] Kaneko T, Hatakeyama R and Sato N 1998 Phys. Rev. Lett. 802602

[18] Takahashi K, Kaneko T and Hatakeyama R 2007 Appl. Phys. Lett. 91261502

[19] Takahashi K, Kaneko T and Hatakeyama R 2008 Phys. Plasmas 15072108

[20] Takahashi K, Kaneko T and Hatakeyama R 2006 Appl. Phys. Lett. 88111503

[21] Takahashi K, Kaneko T and Hatakeyama R 2006 Plasma Sources Sci. Technol. 15495

[22] Takahashi K, Kaneko T and Hatakeyama R 2005 Phys. Rev. Lett. 94215001

[23] Swanson D G 2003 Plasma Waves, 2nd Edition (Institute of Physics Publishing: Bristol and Philadelphia)

[24] Takahashi K, Kaneko T and Hatakeyama R 2005 Phys. Plasmas 12102107

[25] Fukuyama A and Ichida Y 1996 Proc. Int. Conf. Plasma Physics 21342 
(a)

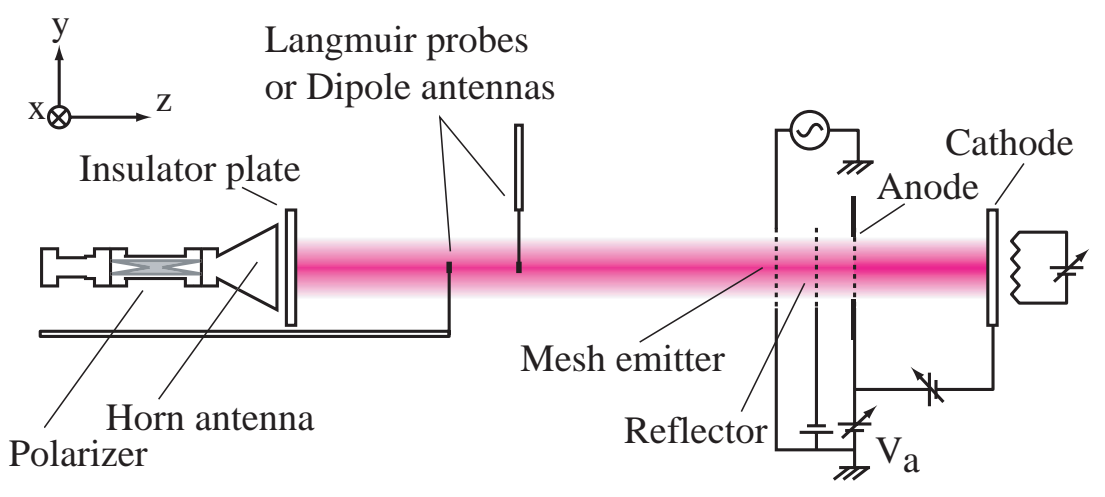

(b)

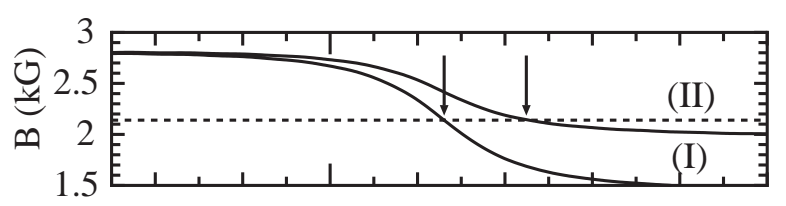

(c)

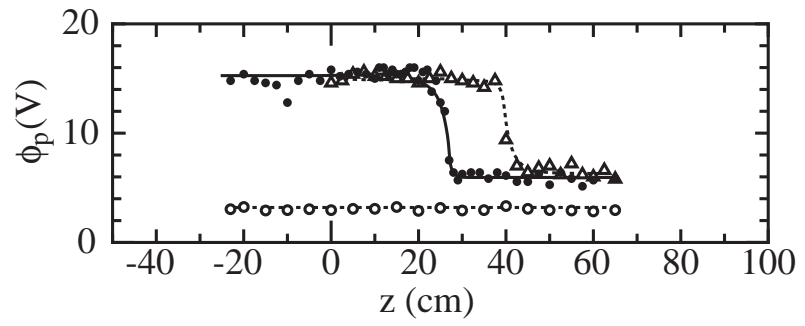

Figure 1. (Color online) (a)Schematic diagram of the experimental setup. (b) Magnetic-field configurations (I) and (II), where the dashed line and the arrows show the magnetic-field strength of ECR and the ECR points. (c) Axial profiles of the plasma potential $\phi_{p}$ for microwave power $P_{i n}=0 \mathrm{~W}$ (open circles) under configuration (I) and for $P_{i n}=50 \mathrm{~W}$ of $m=+1$ mode under configurations (I) (closed circles) and (II) (open triangles). 


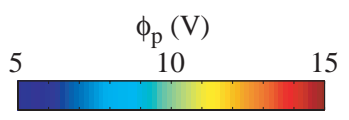

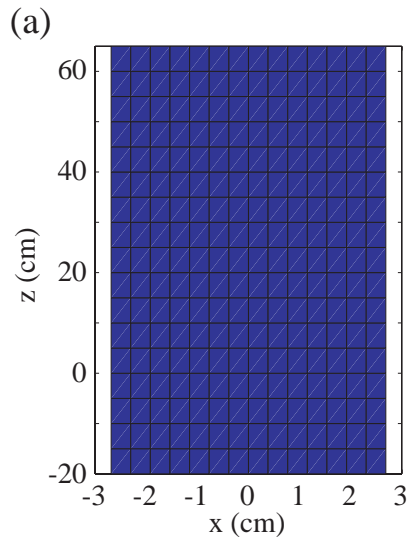

(c)
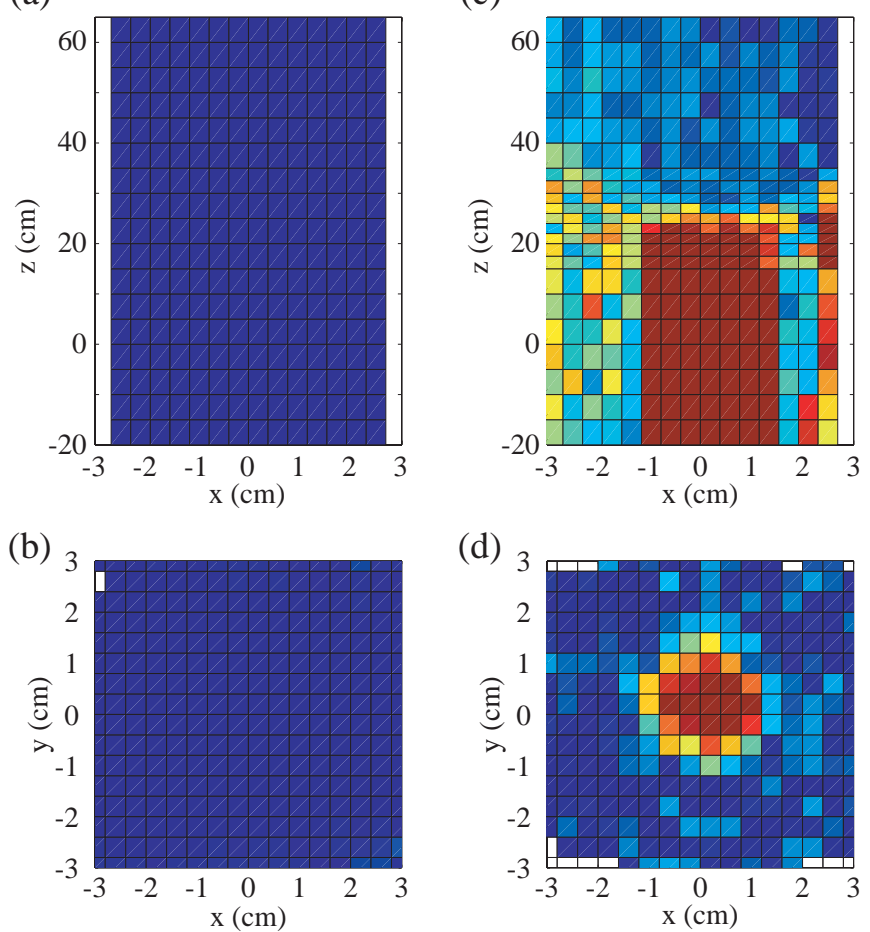

(d)

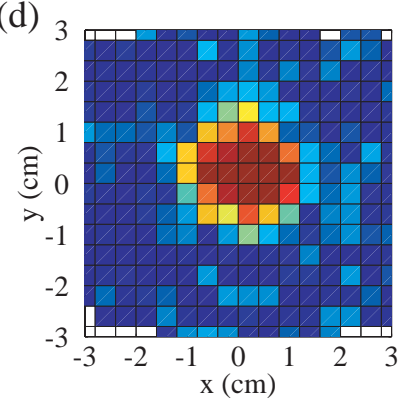

(e)

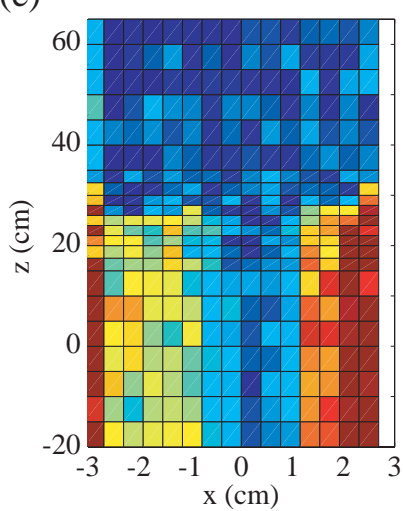

(f)

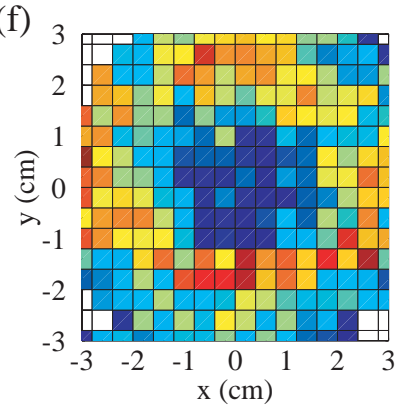

Figure 2. (Color online) $x-z$ and $x-y$ profiles of the plasma potential $\phi_{p}$ for $[(a)$ and (b)] $P_{\text {in }}=0 \mathrm{~W}$, [(c) and (d)] $P_{\text {in }}=50 \mathrm{~W}$ with $m=+1$ mode, and [(e) and (f)] $P_{\text {in }}=50 \mathrm{~W}$ with $m=-1$ mode, where the anode potential is fixed at $V_{a}=15 \mathrm{~V}$. Figs. 2(a), 2(c), and 2(e) are measured at $y=0 \mathrm{~cm}$ under the configuration (I), while Figs. 2(b), 2(d), and 2(f) are obtained at $z=26 \mathrm{~cm}$ under the configuration (II), i.e., the high-potential side. 
(a) $\mathrm{m}=+1$
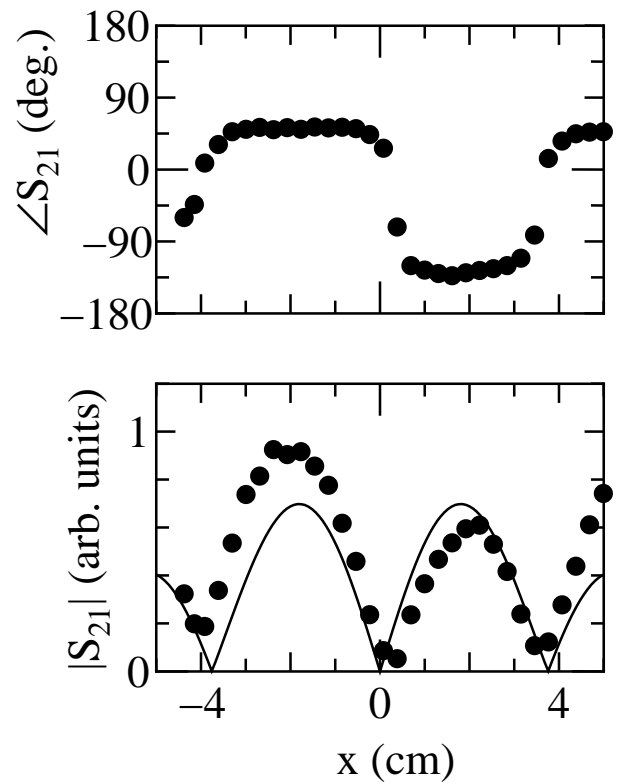

(b) $\mathrm{m}=-1$
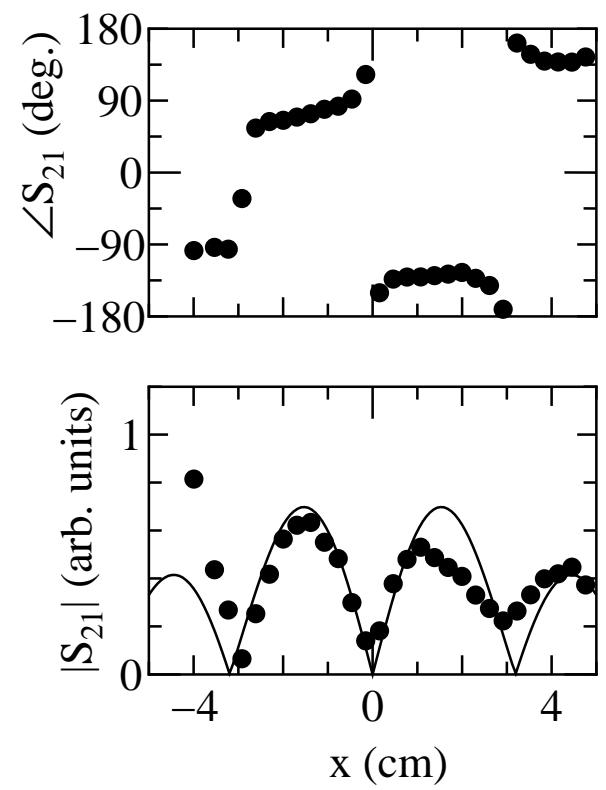

Figure 3. The measured argument $\angle S_{21}$ and absolute value $\left|S_{21}\right|$ of the complex transmission coefficient between the horn antenna and the dipole antenna which is located at $z=15 \mathrm{~cm}$ and detects the axial electric field $E_{z}$, under the configuration (I) for (a) $m=+1$ and (b) $m=-1$ modes. The solid lines show the absolute value of the Bessel functions (a) $J_{1}(1.02 r)$ and (b) $J_{1}(1.20 r)$. 
(a) $\mathrm{m}=+1$

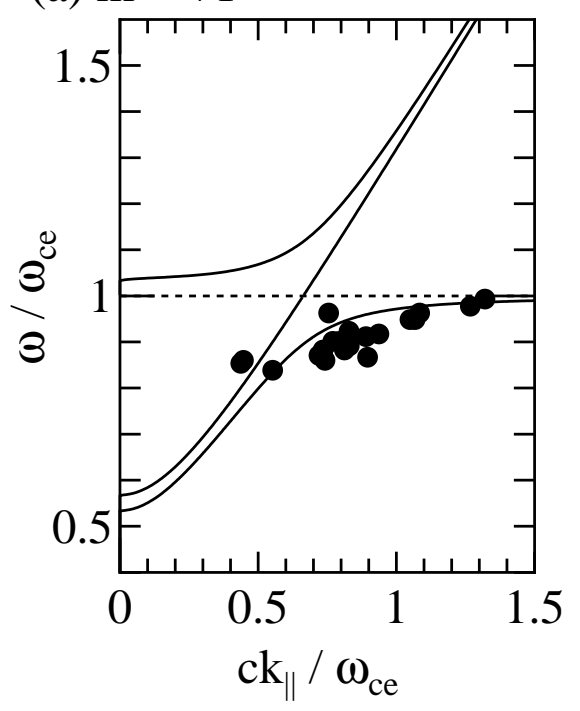

(b) $\mathrm{m}=-1$

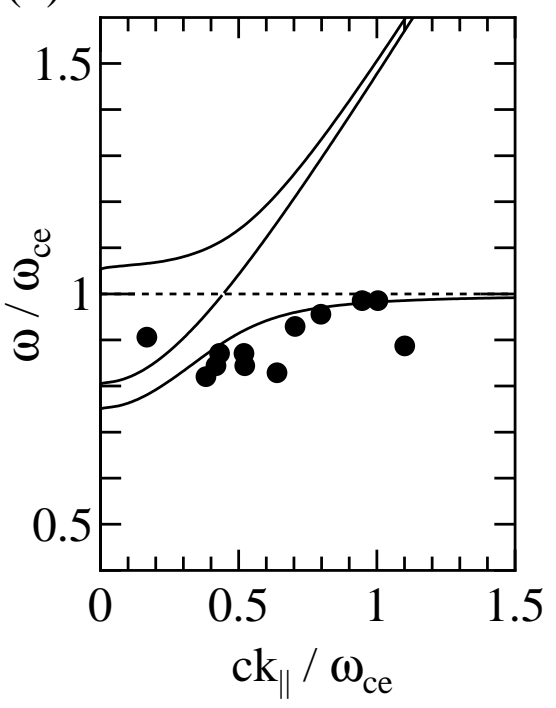

Figure 4. Dispersion relations (solid lines) calculated by Eq. (1) for (a) $k_{\perp}=1.02$ $\mathrm{cm}^{-1}$ and (b) $k_{\perp}=1.20 \mathrm{~cm}^{-1}$, together with the experimentally obtained ones (closed circles) for (a) $m=+1$ and (b) $m=-1$ modes. 
(a) $\mathrm{m}=+1$

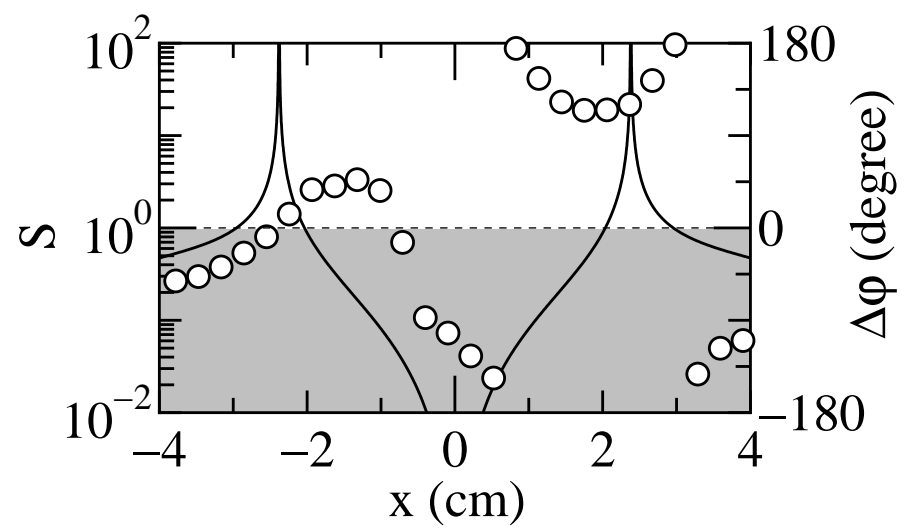

(b) $\mathrm{m}=-1$

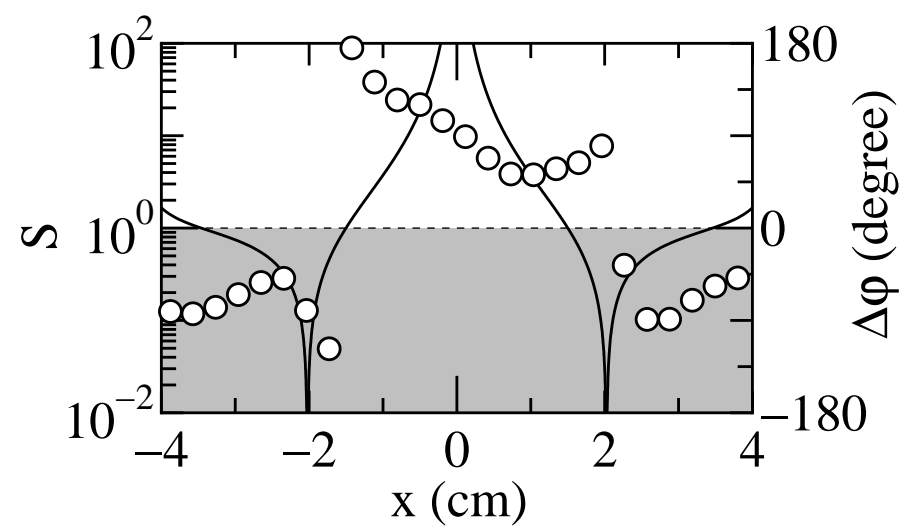

Figure 5. $x$ profiles of the theoretical polarization index $S$ (solid line) and the experimentally obtained phase difference $\Delta \varphi$ between $E_{x}$ and $E_{y}$ of the microwaves for (a) $m=+1$ and (b) $m=-1$ modes at $z=15 \mathrm{~cm}$ under the configuration (I). 
(a)
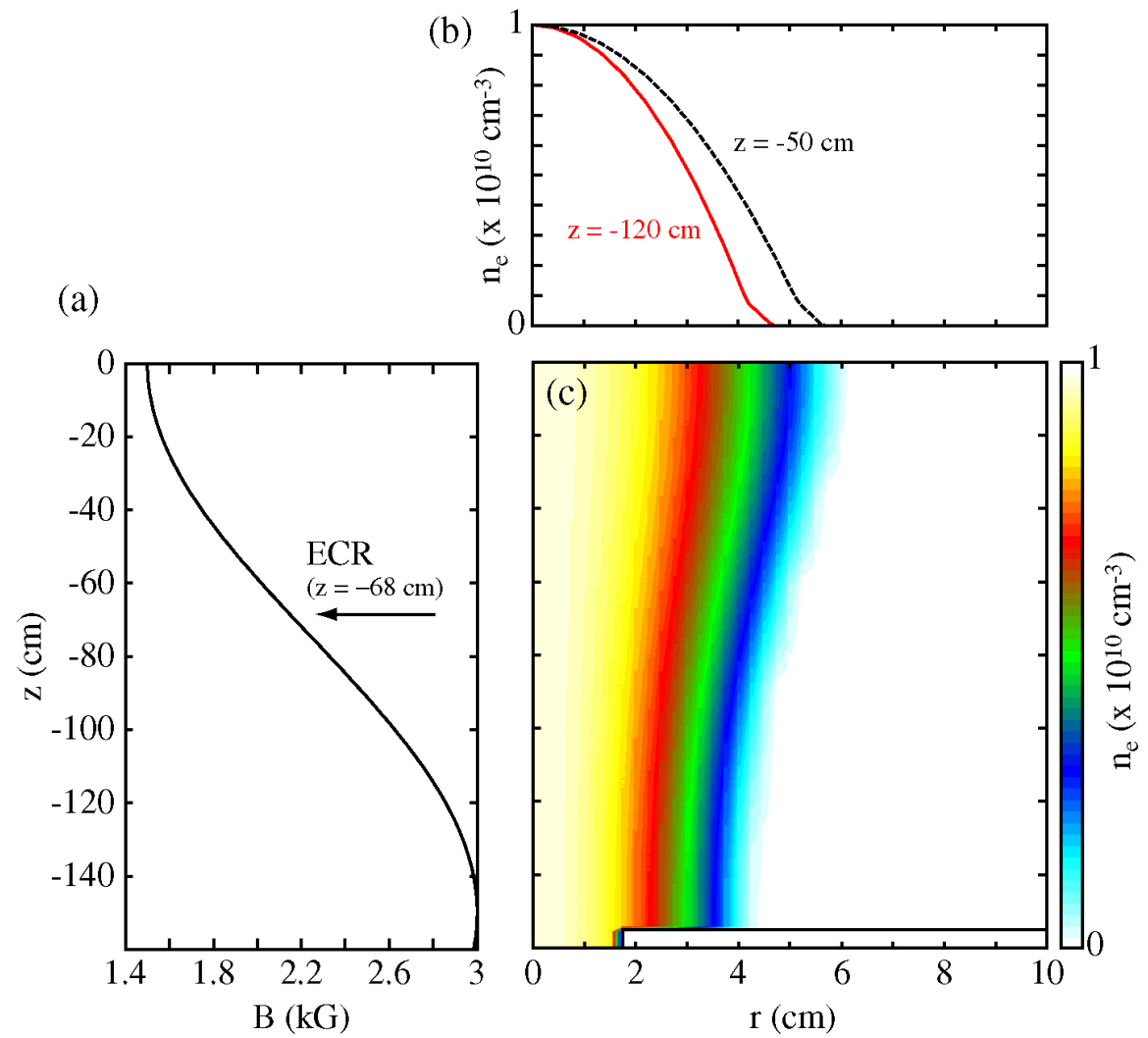

Figure 6. (Color online) Specific profiles used in numerical modeling. (a) Axial profile of the external magnetic field. (b) Radial profiles of the plasma density at $z=-50$ and $-120 \mathrm{~cm}$. (c) $r-z$ profiles of the plasma density. In this configuration, a $1.5-\mathrm{cm}$-radius one-turn loop antenna is located at $z=-159 \mathrm{~cm}$. 

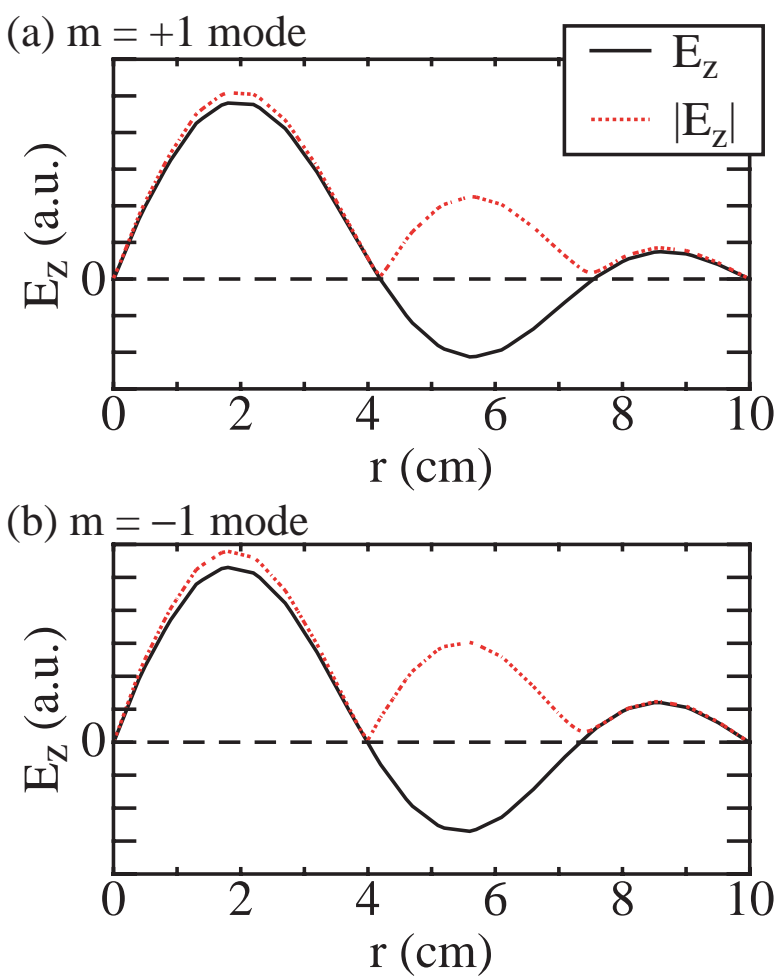

Figure 7. (Color online) Radial profiles of the axial component of the electric field $E_{z}$ at a certain phase and the field strength $\left|E_{z}\right|$ for (a) $m=+1$ and (b) $m=-1$ modes. 


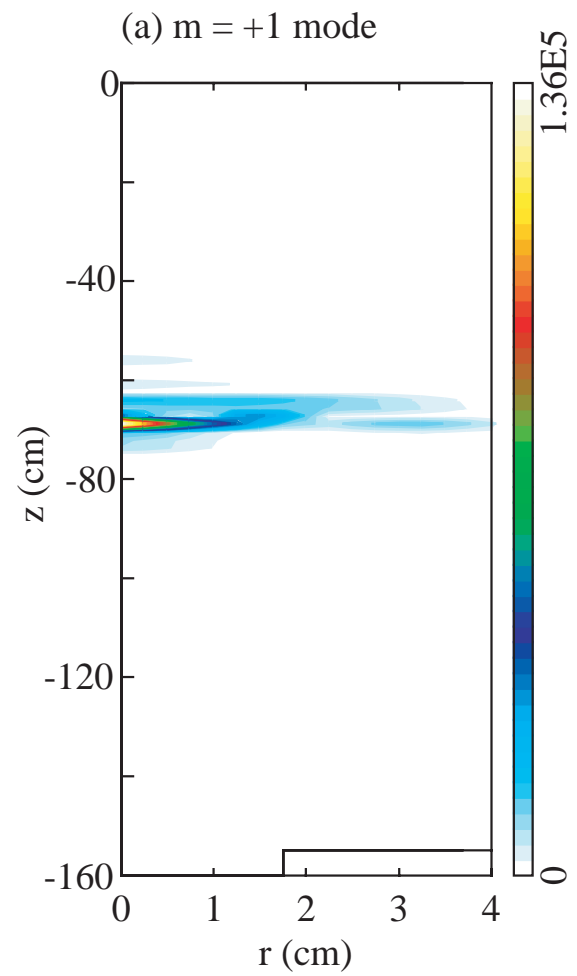

(b) $\mathrm{m}=-1$ mode

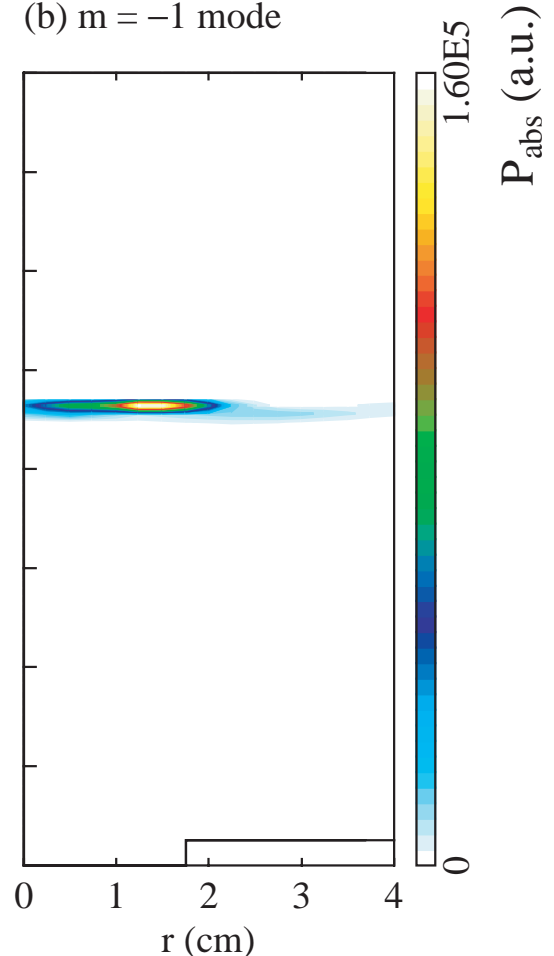

Figure 8. (Color online) $r-z$ profiles of the wave power $P_{a b s}$ absorbed into the plasma for (a) $m=+1$ and (b) $m=-1$ modes. 